\title{
"LE QUALITA TUTTE PERSONALI E TROPPO ARDITE DI QUELLE PITTURE ERANO DI ESEMPIO PERICOLOSO". ALESSANDRO CICCARELLI EN ITALIA Y EL FILOCTETES ABANDONADO (1830-1843)*
}

\author{
"LE QUALITA TUTTE PERSONALI E TROPPO ARDITE DI QUELLE \\ PITTURE ERANO DI ESEMPIO PERICOLOSO". ALESSANDRO \\ CICCARELLI IN ITALY AND THE ABANDONED PHILOCTETES (1830-1843)
}

\section{NOEMI CINELLI ${ }^{* *}$}

Resumen: El artículo quiere aportar nuevos datos sobre el pintor napolitano Alessandro Ciccarelli, en concreto, sobre la etapa italiana de su trayectoria, que coincidió con la formación académica entre Nápoles y Roma, y con la participación en las exposiciones celebradas en ambas ciudades entre 1830 y 1843. Analizaremos la obra Filoctetes abandonado del Museo de Capodimonte, la pondremos en diálogo con la otra versión del MNBA de Santiago de Chile, y demostraremos la procedencia de la inspiración clásica que impregnó sus teorías y sus telas.

Palabras Clave: Alessandro Ciccarelli, pintura decimonónica, Italia, Filoctetes abandonado.

Aвstract: The article provides new data about the Neapolitan painter Alessandro Ciccarelli, in particular about his Italian phase, which coincided with his academic education between Naples and Rome, and with his participation in the exhibitions carried out in both cities between 1830 and 1843. We will analyze the work Abandoned Philoctetes of the Capodimonte Museum in Naples, we will bring it face to face with the other version of the MNBA in Santiago and we will demonstrate the origin from the classic inspiration that permeated his theories and his canvas.

Keywords: Alessandro Ciccarelli, Painting of the 19th century, Italy, Abandoned Philoctetes.

Recibido: 06-03-2019. Aceptado: 04.08.2020.

* El artículo es fruto de las investigaciones en el marco del proyecto Fondecyt iniciación No 11160359 (2016-2019), "Diálogos decimonónicos entre Chile y Europa. La enseñanza del dibujo como vehículo de influjos y transferencias artísticas”, del cual la autora es investigadora principal.

** Doctora en Historia del Arte, con mención Europeo. Académica de la Universidad Autónoma de Chile, Santiago, Chile, y de la Universidad de la Laguna, Tenerife, España. Correo electrónico: ncinelli@ ull.edu.es. Orcid: https://orcid.org/0000-0003-3600-5658 


\title{
1. ENLAZANDO MUNDOS: ALESSANDRO CICCARELLI ¿UN PINTOR CONOCIDO?
}

\begin{abstract}
A lessandro Ciccarelli (Lima, 2010), Alejandro Cicarelli (Zamorano, 2013), Alessandro Ciccarelle (Richter y Valdivieso, 2012), Alexandre Cicarelli (Freire, 1983), Alejandro Cicarelli Mencori (Cruz, 1988; Plath, 2003), Alessandre Ciccarelli Manzoni (Warburg BCI, ad vocem), Alejandro Ciccarelli (Cruz de Amenábar, 2004; De la Maza, 2012). Estas son algunas de las variantes del nombre y apellido del pintor que nos ocupará en las próximas páginas, presentes en documentos de archivo y textos de historiografía artística internacional. Nos referimos a Alessandro Ciccarelli -este es su nombre cierto y escrito correctamente en italiano- artista y docente que nació en Nápoles a principios del siglo XIX y que, en la fecunda época de transferencias culturales que acortaban las distancias entre América y Europa, actuó como propulsor de las políticas educativas gubernamentales en el campo de las Bellas Artes, y como promotor del sistema de enseñanza académica entendido como instrumento para la formación de jóvenes pintores.
\end{abstract}

Su actividad está documentada en tres países: Italia ${ }^{1}$, donde empezó como alumno y pintor; Brasil, donde afinó sus dotes de colorista mientras ejercía como profesor de dibujo en los ambientes imperiales; y Chile, donde alternó la carrera de artista con la de docente. En los años de presencia del pintor, la situación política en estos territorios era respectivamente la siguiente: en Italia soplaban vientos de Risorgimento con Giuseppe Mazzini, Giuseppe Garibaldi y Camillo Benso Conde de Cavour que, alimentados por las notas de las Operas de Giuseppe Verdi, se dirigían hacía la unificación de un estado fragmentado. En Brasil, el Emperador Pedro II de Braganza había sucedido a su padre Pedro I, después de unos años de regencia de su madre María Leopoldina de Austria, inaugurando los años del Segundo Reinado, caracterizados por inestabilidad y continuos balanceos entre las diferentes fuerzas políticas enfrentadas entre sí. En Chile, la lograda Independencia y la República guiada por Manuel Bulnes necesitaban consolidar no solamente sus planes, sino que también la imagen que querían enviar al exterior, y debían empezar a concretar soluciones viables en el campo de las políticas educativas nacionales.

\footnotetext{
${ }^{1}$ Empleamos el término "Italia" para referirnos a un ámbito geográfico y no histórico, pues la Unitá d'Italia en el año 1861.
} 
La cercanía de Ciccarelli a los círculos del Emperador Pedro II en Río de Janeiro (1843-1848) y las actuaciones como director de la Academia de Pintura de Santiago de Chile (1849-1869), han sido los aspectos de su vida que más han atraído la atención de los estudios dedicados a él (Lima, 2012; Ripamonti, 2010; Zamorano, 2013). En particular, la permanencia en el seno de la institución chilena que él mismo inauguró el día 8 de marzo de 1849 , ha sido investigada por la historiografía contemporánea haciendo hincapié en su aparente anacrónica elección del disegno como base del aprendizaje artístico.

Por el contrario, la producción pictórica ligada a los ambientes napolitanos y romanos no ha despertado interés en la crítica y en la historia del arte, lo que conlleva la imposibilidad de concebir un estudio completo y sistemático enteramente dedicado a esta polifacética personalidad que se movió entre dos continentes.

La poca fortuna se debe a lo que Gloria Fossi (2015), en su Dossier Ottocento italiano, define como destino común a toda la pintura de la época de Ciccarelli:

La pintura del siglo XIX (...) no obstante el mercado activo relacionado con un exagerado coleccionismo local, ha sufrido numerosas críticas hasta bien entrada la segunda mitad del siglo XX. Durante mucho tiempo ha surgido la insinuación, cuando no la acusación, de un lenguaje provincial, plagado de titubeos sentimentales, que ni siquiera los pintores más talentosos pudieron evitar de emplear. (p. 4, trad. propia) ${ }^{2}$

Gracias a nuevos datos sobre los años transcurridos en Nápoles y Roma, y a la reflexión sobre la controvertida tela que representa Filoctetes Abandonado, el presente artículo quiere ser una aportación a la etapa italiana de la trayectoria de Ciccarelli, evidentemente la menos conocida, para poder completar la historia de su carrera artística.

\section{CICCARELLI, PINTOR ENTRE NÁPOLES Y ROMA (1830-1843)}

Entre los géneros pictóricos experimentados por Ciccarelli e identificados por Pereira Salas (1992, p. 63), que contemplan pintura religiosa, mitoló-

\footnotetext{
${ }^{2}$ Fuente original: La pittura dell'Ottocento [... nonostante il florido mercato legato a un accanito collezionismo locale, ha patito riserve critiche fin oltre la metá del XX secolo. A lungo ha aleggiato l'insinuazione, quando non l'accusa manifesta, di un linguaggio provinciale, venato di indugi sentimentali, che neppure i pittori di maggior talento avrebbero saputo evitare.
} 
gica y género histórico, es oportuno añadir el retrato y el paisaje, sin que por ello debamos referirnos a este artista como paisajista, pintor de historia o retratista. A raíz de esta consideración, queremos establecer una nueva lectura en la catalogación de su obra, en base a una periodización cronológica -cuyas fechas útiles están marcadas por los viajes emprendidos por el pintor asociada a una localización geográfica- en virtud de sus desplazamientos, que en ningún caso tuvieron trayecto de vuelta:

-Etapa italiana, $1830^{3}-1843$

-Etapa brasileña, 1843-1848

-Etapa chilena ${ }^{4}$, 1848- 1871

La trayectoria artística de Ciccarelli alimentó numerosos debates, algunos culminados en elogios y distinciones, otros en caricaturas y ataques abiertos en la prensa internacional (Cinelli, 2019). Atendiendo a las páginas de los periódicos chilenos más representativos de finales del siglo XIX, consideramos oportuno mantenernos en la misma posición del investigador Zamorano (2013, p. 77), que fija los datos ciertos acerca de la cronología de la biografía de Ciccarelli a partir de lo publicado en dos números consecutivos de la revista El Taller Ilustrado del año I de los días 13 y 20 de diciembre (ver Don Alejandro Ciccarelli 1885(a) y 1885(b).

Durante la etapa italiana de formación, la producción del pintor varía entre copias de grandes maestros del arte italiano, en particular Raffaello Sanzio y Guido Reni, obras de temática mitológica, religiosa, histórica, numerosos retratos, estudios de torsos, árboles y figuras. Aunque en Italia hayamos localizado un restringido número de obras suyas, un escrito de comienzos del siglo XX firmado por Domenico Morelli (1915) ${ }^{5}$, nos ayuda a entender la inspiración clásica en sus telas, que será una constante en su producción nacional e internacional a pesar de los vientos del Romanti-

\footnotetext{
${ }^{3} \mathrm{La}$ fecha coincide con los inicios de los estudios de formación artística en Nápoles. Para noticias sobre el sistema de enseñanza en la Academia napolitana en el siglo XIX, remitimos a la cita de la pág. 135 del presente escrito, en la que queda constancia del orden de los estudios seguido en la institución.

${ }^{4}$ Para definir estas tres etapas, ha sido de fundamental importancia poder contar con la sistematización de una parte de la información, que pudimos recopilar autónomamente efectuando una búsqueda por "Alessandro Ciccarelli", en diferentes portales web dedicados al catastro y difusión del patrimonio artístico: el del Instituto Centrale per il Catalogo e la Documentazione del Ministerio dei Beni Culturali, ICCD, para el caso italiano (http://www.iccd.beniculturali.it consultada el día 8 de febrero de 2020), BRASILIANA ICONOGRAFÍCA para el carioca (https://www.brasilianaiconografica.art.br consultada el día 8 de febrero de 2020) y SURDOC para el ámbito chileno (http://www.surdoc.cl, consultada el día 8 de febrero de 2020).

${ }^{5}$ Domenico Morelli fue el artista que, junto con Filippo Palizzi, se considera el más representativo de la pintura napolitana de siglo XIX. Muy cercano a los ambientes frecuentados por Ciccarelli, director de la Accademia di Belle Arti de Napoles, se movió entre inspiraciones románticas y veristas, tomando parte en los debates florentinos de los Macchiaioli sobre el Realismo en la pintura decimonónica.
} 
cismo que soplaban con fuerza desde el Norte de Italia (Lorenzetti, 1953). Recordemos que la juventud de Ciccarelli transcurrió entre la Ciudad Partenopea y la Ciudad Eterna, donde el lenguaje y las normas clásicas representaban, desde hacía siglos, la base inamovible del sistema de producción y creación artística:

Todavía el ambiente italiano estaba saturado de clasicismo: la Mitología y los Héroes de Homero se habían adueñado de él. Hayez pintaba, a la manera de Appiani y de Sabatelli, sujetos mitológicos: el grande Alfieri iba de la mano del divino Foscolo, preparando a Giacomo Leopardi para la fusión de la forma clásica con los sentimientos más reales e íntimos. Pero, aunque Italia tenía grandes artistas, no era ella la que debía empezar el movimiento vertiginoso del arte del corazón, del arte romántico, del arte verdadero. Mientras que nosotros nos especializábamos como maestros del arte clásico, que aquí llaman "imperial", desde el Norte se desencadenaba una tempestad, que nosotros percibíamos con retraso, como se percibe el trueno lejano cuando el relámpago estalla a una distancia sin fin; poquísimos entre nosotros, percibieron el olor de la tormenta. (Morelli, 1915, pp. 74-75, trad. propia) ${ }^{6}$

Gracias al Centro di Documentazione del Museo di Capodimonte ${ }^{7}$ hemos recopilado las obras que Ciccarelli expuso, el año y el lugar donde se pudieron admirar. Reportamos las informaciones hasta ahora inéditas:

1830

-Como alumno en el Real Museo Borbónico, Nápoles:

- Apolo Pastor en la campaña - Óleo

-En el Real Instituto de Bellas Artes de Nápoles:

- Retrato de hombre - Dibujo

\footnotetext{
${ }^{6}$ Fuente original: Ancora l'ambiente era in Italia saturo di classicismo: la Mitologia e gli Eroi di Omero ne avevano preso possesso. L'Hayez dipingeva, nel genere dell'Appiani e del Sabatelli, soggetti mitologici: il grande Alfieri signoreggiava col divino Foscolo, preparando a Giacomo Leopardi la fusione della forma classica coi sentimenti più reali e più intimi. Ma, sebbene l'Italia avesse il vanto di così grandi artisti, pure non era essa che doveva iniziare il vertiginoso movimento dell'arte del cuore, dell'arte romantica, dell'arte vera. Intanto che noi eravamo maestri dell'arte classica, che qui chiamiamo «imperiale», dal Nord si scatenava una tempesta, che noi risentivamo con ritardo, come si sente il tuono lontano quando il fulmine scoppia a sterminata distanza; pochissimi, presso di noi, fiutarono lodore del temporale (tda).

${ }^{7}$ Queremos expresar nuestros agradecimientos a Marco Liberato, del Dipartimento di Documentazione del Museo e Real Bosco di Capodimonte di Napoli, por las informaciones proporcionadas con fecha $29 / 11 / 2018$.
} 
1833

-En el Real Instituto de Bellas Artes de Nápoles:

- Retrato viril a media figura

- Santa Cecilia copiada del original de Guido Reni

- Dante y Virgilio a las puertas del infierno

- Resurrección de Cristo

- Academia de torso de espalda

- San Sebastián

- Guerrero herido

- Busto de un viejo barbudo tomado del natural

- La prédica de San Juan Evangelista en el desierto

- Retrato del Caballero Don Giuseppe Forcella a media figura

- Retrato de un joven a media figura

1835

-Como alumno del Real Instituto de Bellas Artes de Nápoles, ya pensionado en Roma:

- Muerte de Arquímedes

1837

-Como alumno del Real Instituto de Bellas Artes de Nápoles, aún pensionado en Roma:

- Filoctetes herido

- Coronación de María Santísima con Cristo y ángeles

- copia de Rafaelo Sanzio

1839

-Como alumno del Real Instituto de Bellas Artes de Nápoles, aún pensionado en Roma:

- Telémaco y Termosiri

- Francisco I Re de Francia en la Batalla de Pavia

1841

-Como alumno del Real Instituto de Bellas Artes de Nápoles, aún pensionado en Roma:

Su Majestad el Rey N.S., en compañía de S.A I. Archiduque Carlos y seguido por Generales, pasa en reseña las tropas del campo

1843

-Como alumno del Real Instituto de Bellas Artes de Nápoles, aún pensionado en Roma:

- Retrato masculino a media figura. 


\section{EL FILOCTETES ABANDONADO: UNA OBRA COMPLEJA}

Como relatan con casi un siglo de diferencia Álvarez Urquieta (1928, p. 25) y Zamorano (2013, p.78), la etapa italiana de Ciccarelli fue muy fructífera, como demuestran las numerosas distinciones recibidas: la Medalla de Plata en la Exposición de Nápoles, en 1833; el Segundo Premio Mayor con Medalla de Oro en 1839 en Roma, por sus obras Filoctetes abandonado y Telémaco y Termosiri, y el Gran Premio con Medalla de Oro de Primera Clase en la Exposición de Nápoles de 1841.

En particular, el Filoctetes ha sido una de las pinturas que más ha alimentado el debate de la historiografía contemporánea. En los Annali Civili del Regno delle Due Sicilie de 1837 (ver Accademia delle Belle Arti di Napoli, 1837) que relatan el concurso en el cual fue expuesta la obra, es posible remontar al texto que los alumnos tenían como referencia para el desarrollo de la composición:

Sentado en el acantilado extremo de una roca, el hijo de Peante, casi desnudo, que solo está parcialmente cubierto con una capa roja corta, y extiende su brazo izquierdo con la mano abierta hacia el mar, como un hombre rendido por el asombro y la indecible angustia.

... Oh, hijo, ¿cómo crees que me quedé cuando me desperté y todos me habían dejado? ¡Oh, qué lamento fue el mío! Oh que aullidos los míos, cuando vi los barcos con los que había llegado, desapareciendo, y aquí nadie me socorre ni ofrece alivio a mi dolor; y miro a mi alrededor, y ya no veo más que tristeza, igran tristeza! (p. 137, trad. propia) ${ }^{8}$

Se trataba de un fragmento de la obra de Sófocles, Filoctetes, tragedia representada por primera vez en 409 a. C. en el Teatro de Dionisio en Atenas con motivo de las festividades dedicadas al dios y que, a su vez, se inspiraba en el mito recopilado en la Ilias Parva.

El protagonista sofocleo -detentor del arco que habría podido hacer capitular Troya, abandonado por sus compañeros en la isla de Lemnos y héroe que apiadó a Neoptólemo- estimuló la fantasía de artistas que, entre el Renacimiento y el Ottocento italiano, dejaron varias representaciones de los episodios de su vida.

\footnotetext{
${ }^{8}$ Fuente original: Siede sulla estrema balza d'uno scoglio il figliuol di Peante, quasi nudo, chè solo in parte lo copre breve mantello di color rosso, e distende il braccio sinistro con la mano aperta verso il mare, como uomo soprarreso da stupore ed angoscia indicibile.

(...) O figlio, qual pensi tu ch'io rimanessi, quando partiti tutti, mi levai dal sonno? Oh, che piangere fu il mio! Quali ululati furono i miei, quando tutte sparite mirai le navi, onde venutio io m’era, e quà nessun che mi socorra e porga al mio dolor sollievo; e intorno mandar lo sguardo e non veder per tutto fuorchè mestizia, alta mestizia.
} 
El Metropolitan Museum of Art de Nueva York guarda un lekythos en terracota, procedente del Ática y fechado el 420 a. C., que desde 1879 a 1884 formó parte del grupo de cerámicas de figuras rojas de la Colección Alessandro Castellani (Fig. 1). Creemos que esta pequeña obra haya sido una de las fuentes primarias para las sucesivas iconografías del héroe en Lemnos. En la vasija sin atribuir, objeto destinado a contener aceites y ungüentos perfumados, resulta curiosa la elección del tema para la decoración, ya que el abandono de Filoctetes, más que por los lamentos causados por el dolor de la herida, se debía al tremebundo olor que desprendía de esta y que sus compañeros no soportaban. En la parte delantera de la diminuta obra, podemos admirar un joven elegante y barbudo sentado en una roca debajo de un árbol, con el carcaj apoyado en el suelo, mientras se cura la herida fruto de la mordedura de una víbora en el pie. Su cuerpo viste un quitón corto sin mangas y su desolación es evidente en la inclinación de la cabeza.

Siglos más tarde, estimulado quizás por la editio princeps de las tragedias de Sófocles publicada en Venecia en 1502 por Aldo Manucio, el escultor autóctono Antonio Lombardo se dedicó casi al final de su vida -entre los años 1510-1515- a una interpretación del mismo tema (Fig. 2), realizando un relieve en mármol blanco con incrustaciones en mármoles rojos y purpúreos, conservado hoy en el Victoria \& Albert Museum.

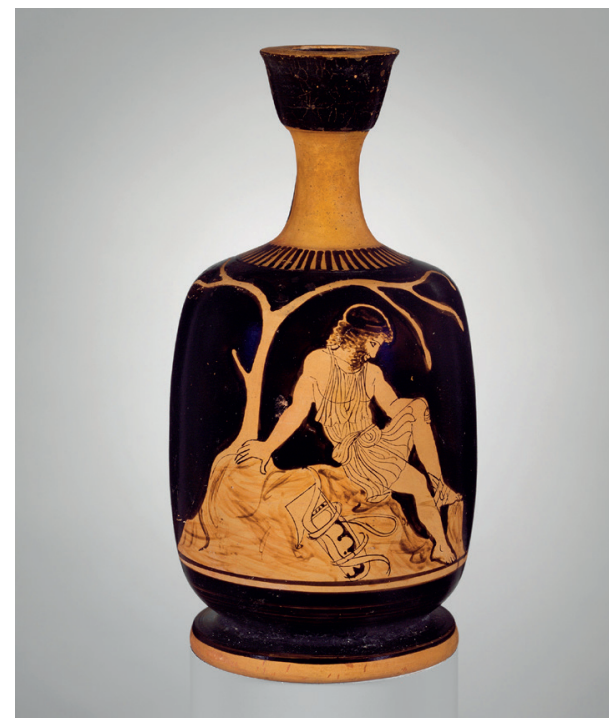

Figura 1. Filoctetes en Lemnos, Terracota a figuras rojas, ca 420 a.C., 15,7 x $7 \mathrm{~cm}$, Metropolitan Museum of Art de Nueva York. Num.Inv. 56.171.58. 


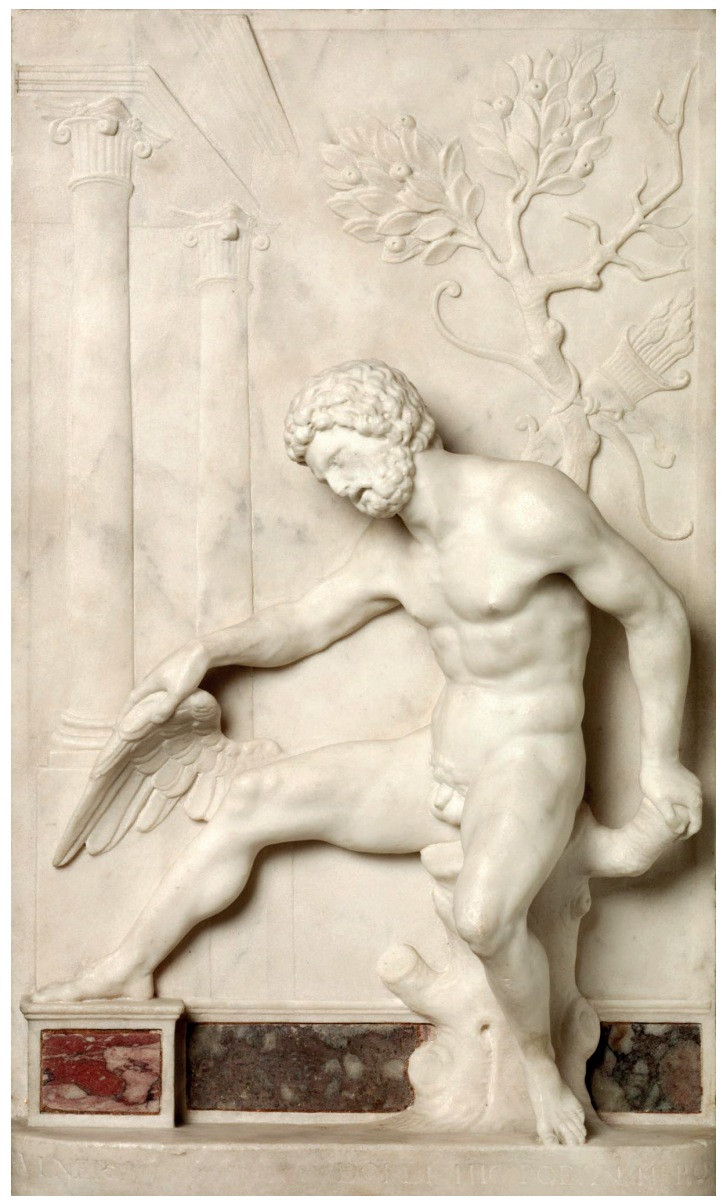

Figura 2. Filoctetes en Lemnos, Relieve en mármol blanco, rojo y púrpura, ca 1510-1515, 41,3×25x9,5 cm, Victoria \& Albert Museum, Londres, Num.Inv. A.9-1928.

La atmósfera desoladora que impregna la vasija del Metropolitan se pierde en la composición de Lombardo, para dejar espacio a un ensayo admirable sobre el desnudo masculino, cuyos cánones inspirados en los modelos de la Grecia Clásica resultan evidentes en la curva serpentina de reminiscencia praxiteliana, en el tratamiento del pliegue inguinal y en la musculatura del torso. Sin embargo, son innegables las similitudes entre las dos obras, especialmente en la pose del cuerpo y de la cabeza. En la composición del Victoria \& Albert Museum, gracias al gesto del arquero 
que se abanica la herida con el ala de un pájaro recién cazado, resulta clara la sensación de aire circulando en la representación. El dolor de Filoctetes es patente en la tensión del brazo izquierdo apoyado en la rama robusta del árbol.

En el caso de la tela de Ciccarelli, se trata de su obra mitológica más conocida, a la que se dedicó en dos ocasiones: la primera, ya citada y realizada en 1837, es una tela al óleo de grandes dimensiones que se guarda en el Museo di Capodimonte de Nápoles (Fig. 3); la segunda, es obra conocida en Chile gracias a la puesta en valor del Museo Nacional de Bellas Artes (MNBA) de Santiago en ocasión de la exposición En-Clave Masculino, celebrada en 2016 (Fig. 4).

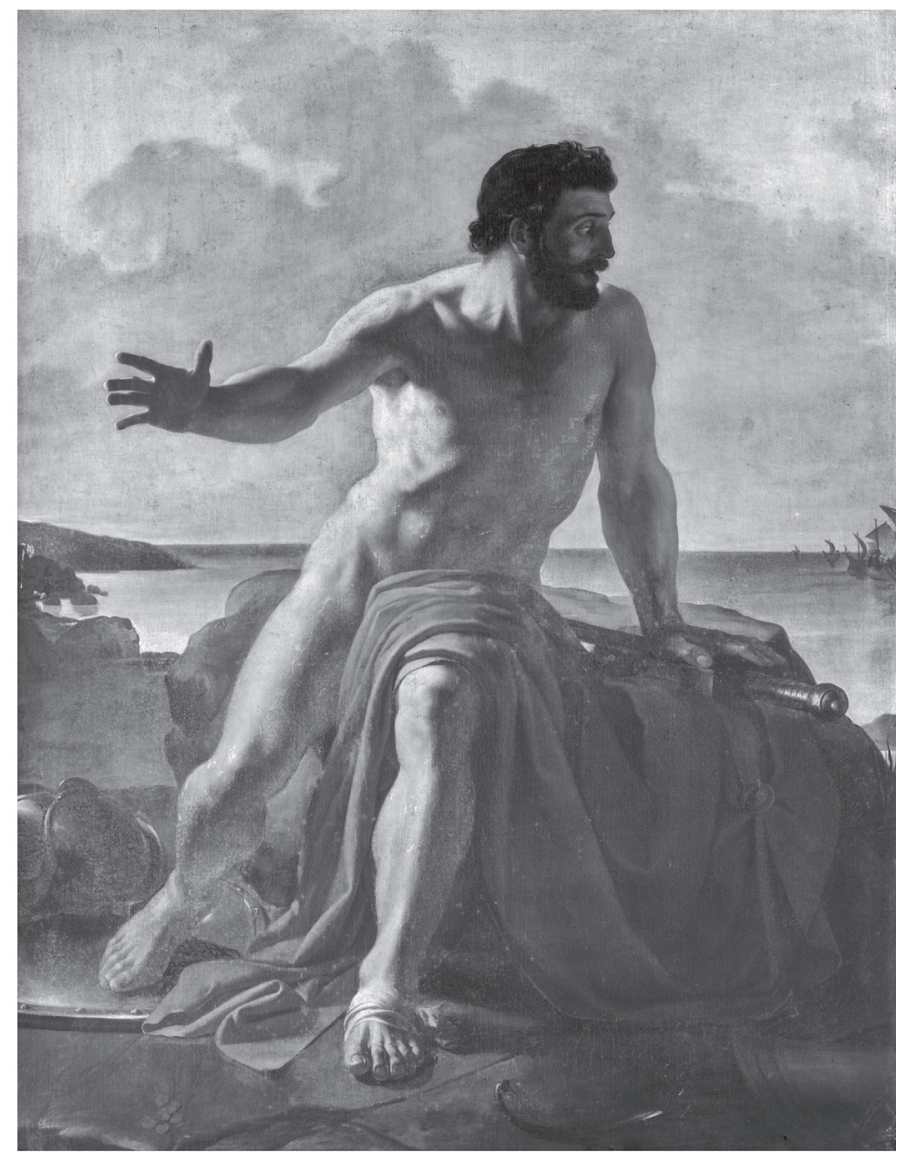

Figura 3. Filoctetes herido al pie, óleo sobre tela, 250x160cm, Museo di Capodimonte de Nápoles, Num.Inv. IC3925. 


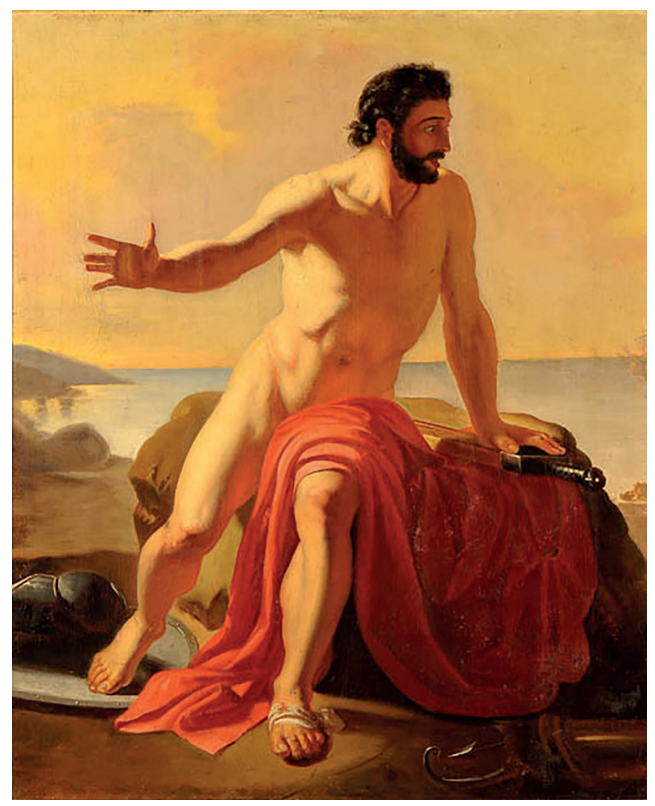

Figura 4. Filoctetes abandonado, óleo sobre tela, 75x62 cm, MNBA de Santiago, Num. Inv.PCH-0107.

Fijándonos en la posición de la parte inferior del cuerpo de Filoctetes, podemos sostener que Ciccarelli pintó su obra influenciado por la de Lombardo, a su vez plasmada sobre la vasija citada. Aún no hemos podido averiguar de qué manera el pintor napolitano pudo llegar a esta fuente, si fue a través de un grabado o gracias a otros autores inmediatamente anteriores a él, que se inspiraron en ella. Nos referimos en este último caso a Francisco Hayez, cuya versión de Filoctetes (Fig. 5), fechable entre 1818-1820, recuerda el relieve lombardiano en cuanto a composición y escenografía. En esta pintura, como subrayó Poppi (1996), "la búsqueda de una renovación cromática estudiada gracias a los ejemplos de la tradición véneta, confirmaría la ejecución de la obra en los años en los que Hayez pone en práctica la clase aprendida en Roma" (pp. 201-202, tda). En el dibujo preparatorio (Fig. 6) es evidente el contacto que Hayez mantiene con el Torso del Belvedere y con las esculturas de Michelangelo Buonarroti; en concreto, con las personificaciones del Día y de la Noche de la Tumba de Giuliano de Medici en la capilla florentina. 


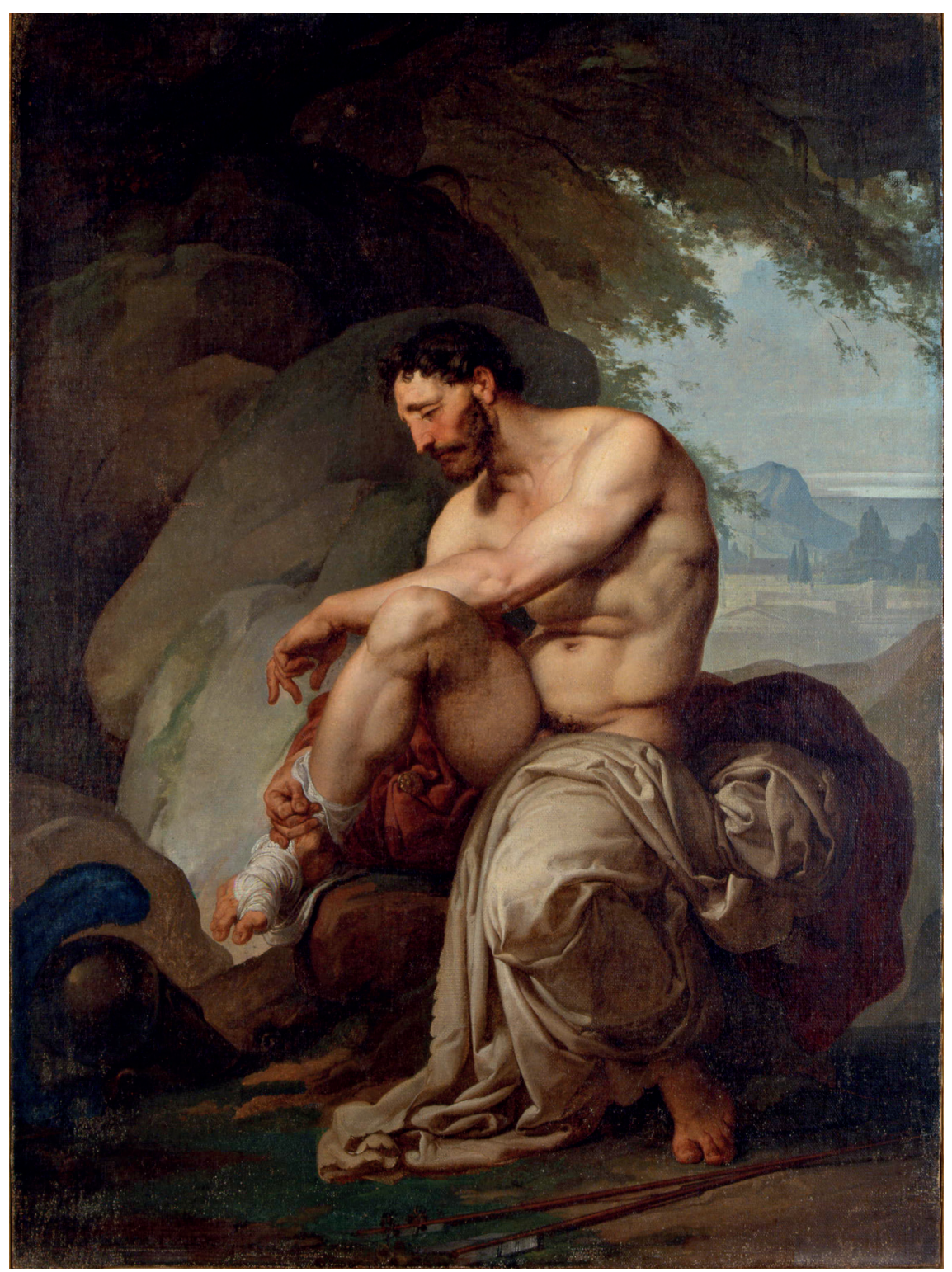

Figura 5. Filoctetes herido, óleo sobre tela, ca. 1818-1820, 125x93cm, Museo d'Arte Moderna di Bologna, Num. Inv. N.P. 


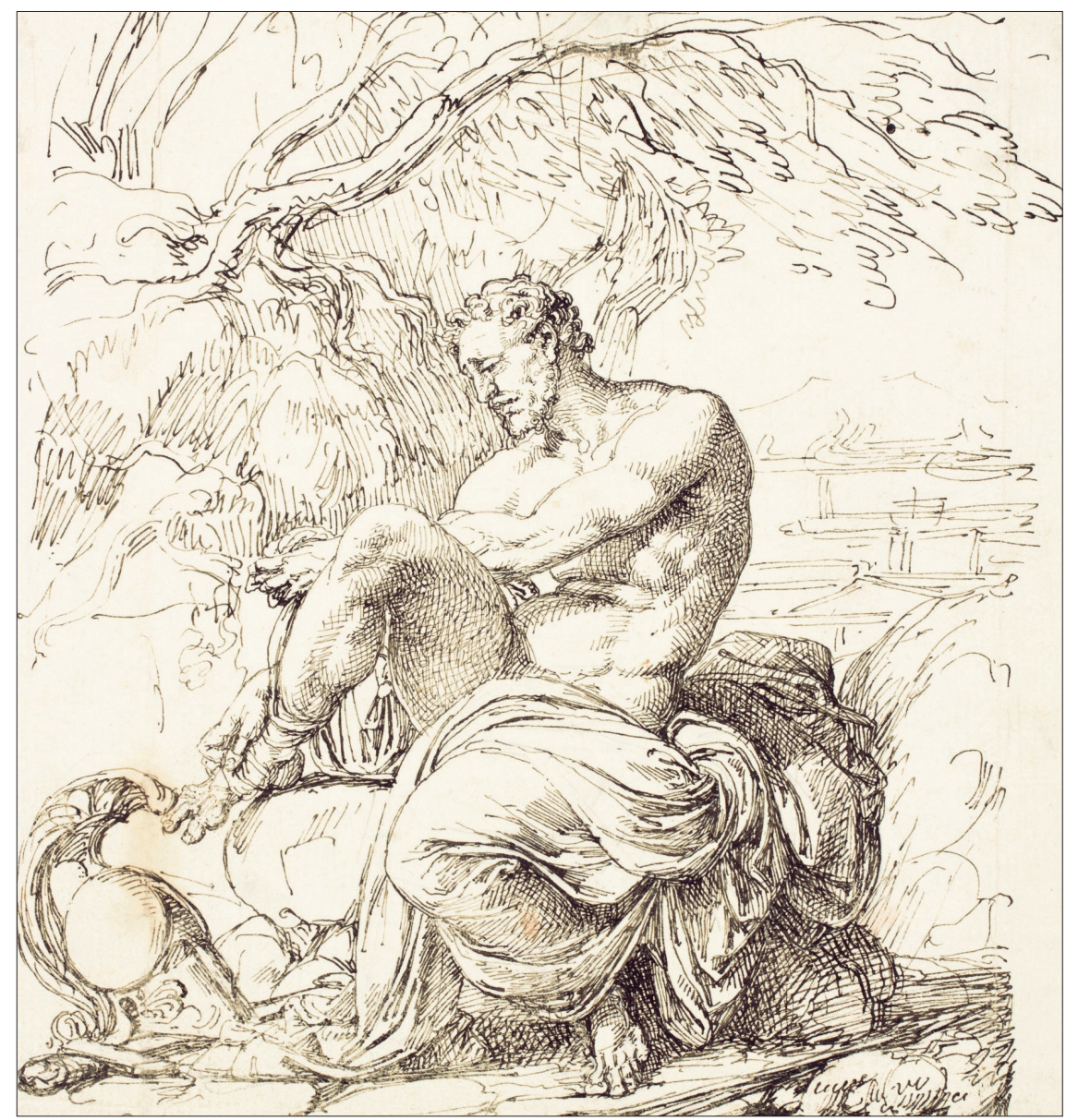

Figura 6. Filoctetes herido, dibujo sobre papel con pluma y tinta marrón oscuro, sobre trazas de tiza negra; 21,19 cm, Colección Sotheby, en Old Master Drawings, Num. Inv. N.P.

Por lo que a la gran tela ciccarelliana se refiere, desde su primera exposición en Italia, la prensa artística de la época y los estudios posteriores han reconocido en ella una obra de grandes cualidades estéticas y técnicas. Algunos elogios fueron reportados en periódicos romanos, toscanos, turineses y otros, como el que proponemos a continuación, en los citados Annali Civili del Regno delle Due Sicilie, relativos al año 1837: 
Mucha vitalidad y expresión están en la cabeza del héroe; así como es bien ajustado el movimiento de toda su persona. Sin embargo, el diseño se inclina hacia lo real y lo torpe, en la espacialidad de las piernas y en los pies: un defecto que es más claro al ojo porque se trata de dimensiones colosales y gigantescas. Parece que en esta obra se deseaba una cierta singularidad en los efectos de luz: pero en medio de un fondo muy claro y vaporoso, la luz se refleja en los hombros del guerrero, de modo que él permanece en la sombra. A alguien no le gustó (...) pero nosotros diremos abiertamente que, incluso de esta manera, puede hacerse una hermosa pintura. Y de verdadero en el Filoctetes vislumbrarás esa repercusión de la luz y entre los tonos un acuerdo muy armonioso. Quisiéramos recomendarle a Ciccarelli que comience bien lo que otros han hecho mal, es decir, que no pierda nunca de vista el hermoso cuadro de Rafael, del cual hizo una copia digna de elogios. (Accademia delle Belle Arti di Napoli, 1837, p. 137, trad. propia) ${ }^{9}$

Años más tarde sigue el favor del público, como se lee en la revista L’Arte in Italia. Rivista mensile di Belle Arti: "Mayor ingenio demostró Alessandro Ciccarelli, quien en 1838, como ensayo de pensionado exhibió al herido Filoctetes, en el que hizo destacar inmediatamente su individualidad, gracias a la manera tan personal de pintar" (Pateras, 1872, p. 118, trad. propia) ${ }^{10}$.

Así fue como se expresó Eduardo Dalbono (ver 1915) el 25 de noviembre de 1901, en ocasión de la Commemorazione letta alla Real Accademia di Archeologia, Lettere e Belle Arti di Napoli, contribución recogida por Benedetto Croce en 1915:

Fijémonos en las composiciones de Mancinelli y de Ciccarelli (Pinacoteca di Capodimonte). En el primer (Ulysses que lanza el disco), un diseño franco, ágil y animado, con un color sobrio y armonioso, hace creer que la paleta realmente sirvió de algo. El otro (el Filoctetes), menos verdadero y más

\footnotetext{
${ }^{9}$ Fuente original: Molta vivezza ed espressione é nella testa dell'eroe; come ben aggiustata é la movenza di tutta la sua persona. Il disegno, per altro incline al vero ed al goffo, in ispazialitá per le gambie e pe 'piedi: difetto che piú corre all'occhio perché trattasi di forme colossali e gigantesche. Sembra che in questo lavoro siesi vagheggiata una certa singolaritá negli effetti di luce: perocché in mezzo al fondo assai chiaro e vaporoso la luce riflette sulle spalle del guerriero sí che questi rimane in ombra. Spiaceva a taluni [...] noi apertamente diremo che anche in tal modo puó farsi una bella pintura. E di vero nel Filottete scorgerai con garbo quel ripercuotere della luce, e sparso nelle tinte un accordo assai giudizioso. Ben vogliamo raccomandare al Ciccarelli che imitasse nel bene ció che altri ha fatto nel male: chè quanto dire, che non perdesse di mira il bellissimo dipinto di Raffaello, del cuale ha pur fatto una copia degna di lode (tda).

${ }^{10}$ Fuente original: Maggiore ingegno sortí Alessandro Ciccarelli, il quale nel 1838 per saggio di pensionato espose il Filottete ferito, nel quale fece súbito spiccare la sua individualitá per una maniera tutta propia.
} 
académico en el movimiento, pero de una vivacidad de colores y una luz tan deslumbrante, como si se tratara de una figura que está verdaderamente al aire libre. (p. 74, trad. propia) $)^{11}$

A las reseñas positivas de las revistas Il Tiberino y La Pallade, se unieron las de Il Telescopio y La Rivista Nacional.

Resulta entonces discrepante pensar en la versión del Filoctetes del MNBA de Santiago de Chile como una obra mal lograda y criticada por los chilenos contemporáneos de Ciccarelli y por los estudiosos del siglo siguiente. Llama la atención la disparidad de juicios expresados por la historiografía artística en Italia y en Chile acerca de las dos versiones conocidas.

Pedro Lira (1902) fue lapidario en este sentido cuando escribió: “(...) En Chile produjo Ciccarelli muy pocos cuadros originales, pero sí muchos retratos. Obras: Filoctetes, Batalla de Pavía..." (ad vocem). Antonio Romera (1951, p. 69), Ricardo Bíndis (1985, p. 38), Galaz e Ivelic (2009, p.74) contribuyeron también a alimentar los juicios negativos alrededor de Ciccarelli y su Filoctetes.

La pequeña obra santiaguina, aún por fechar, se ha considerado un no acabado debido a sus reducidas dimensiones, al tratamiento semiesquemático del dibujo, a la aplicación de altos contrastes y a la restringida gama cromática de la paleta (De La Maza, 2016, p.119).

Si la obra fue realizada en Italia, sería anterior a 1843, año del viaje de Ciccarelli a Brasil; es más, podríamos pensar que fue un ejercicio relacionado con la Exposición de 1839, y que por el significado que podría encerrar para el artista ganador de un Premio Roma, Ciccarelli quizás haya decidido traer consigo la diminuta tela a América. "El dibujo es la gramática del arte" (Cruz de Amenábar, 2004, p. 100), decía el pintor, así que viajaba con el dibujo y dejaba la tela en Italia.

Si la obra fue realizada en América antes de 1858, como De la Maza propone en el catálogo del MNBA (2016, p. 119), es probable que Ciccarelli la realizara como ejercicio académico sobre dibujo anatómico.

Nosotros apostamos más por la primera hipótesis debido a detalles como el pequeño escorzo de marina a la derecha del cuadro, el cielo amarillento y, sobre todo, por la minucia en el tratamiento pictórico reservado al arma de Filoctetes y a su materialidad metálica.

\footnotetext{
${ }^{11}$ Fuente original: Guardiamo i concorsi di Mancinelli e Ciccarelli (Pinacoteca di Capodimonte). Nel primo [Ulisse che lancia il disco), un disegno franco, flessuoso, movimentato, con colore sobrio ed armonioso, da far credere che la tavolozza servisse davvero a qualche cosa. Laltro (il Filottete), meno vero e più accademico nel movimento, ma di una vivacità di tinte e di una luce così smagliante, come una figura che si trovi veramente all'aria aperta.
} 
Creemos que la datación incierta de creación de esta segunda versión juegue a favor de Ciccarelli, al abrir varias posibilidades en cuanto a su lectura. ¿Fue un estudio preparatorio para el concurso napolitano? ¿ $\mathrm{O}$ una interpretación íntima y personal de un tema tan querido como el que le valió reconocimientos en su patria natal? ¿O quizás, fue un ejercicio para sus alumnos americanos a los que dar prueba de la complejidad de la composición de un ensayo anatómico del cuerpo masculino? Las tres posibilidades serían plausibles y nos hablarían de tres facetas diferentes del pintor: el brillante alumno en formación, el amante de la añorada Antigüedad Clásica dejada en Italia, o el profesor que enseña a sus discípulos chilenos gracias a una perfecta síntesis entre teoría del arte y creación.

Queremos concluir este apartado con dos citas, la primera, para poner la historiografía artística frente a un necesario replanteamiento crítico de los juicios expresados sobre Ciccarelli, especialmente en Chile en el siglo $\mathrm{XX}$, y que han generado la imagen de un pintor y profesor muy poco en línea con el medio artístico al que llegó. Se trata de las palabras del ya citado Domenico Morelli (1915) que escribe sobre las Exposiciones Bienales de Nápoles, reflexionando sobre el interés despertado por la obra de Ciccarelli pensionado:

Las obras que casi siempre hacían los honores de la exposición fueron los "ensayos", que enviaban desde Roma nuestros pensionados. El autor de la mejor y más importante obra de pintura, escultura y arquitectura era considerado merecedor de la gran medalla de oro, y su nombre era acuñado con las palabras "premio al mérito", y esta distinción en ese momento tenía un gran valor, no solo en la opinión de los ciudadanos, sino también en todo el Reino. (...) Ciccarelli envió el Filoctetes y el Telémaco y Termosiris que canta debajo de un bellísimo plátano iluminado por el sol (...) A nosotros jóvenes nos decían que, entoces, las cualidades demasiado personales y osadas de aquellas pinturas, eran un peligroso ejemplo. (p. 6 , trad. propia) ${ }^{12}$

La segunda, para desmitificar su anacrónico impulso dado al "dibujo como gramática del arte", natural posición que se explica con la educación

\footnotetext{
${ }^{12}$ Fuente original: Le opere che facevano quasi sempre lonore della Mostra erano i «saggi», che mandavano da Roma i nostri pensionati. Lautore dell'opera migliore e più importante di pittura, di scultura e di architettura, era giudicato degno della grande medaglia d'oro, e il suo nome veniva contrassegnato conia scritta: "premio al merito distinto», e questa distinzione aveva allora un gran valore, non solo nella opinione dei concittadini, ma anche in tutto il Regno [...] Il Ciccarelli mandò il Filottete ed il Telemaco e Termosiri che canta sotto una bellissimo platano illuminato dal solo [...] A noi giovani si diceva, allo, che le qualitá tutte personali e troppo ardite di quelle pinture erano di esempio pericoloso.
} 
artística recibida durante su etapa italiana. Una vez más, es Morelli (1915) quien escribe:

El orden de nuestros estudios, entonces, era el mismo que se usa ahora: del grabado pasamos al relieve, y de la estatua al desnudo y la pintura. Había concursos mensuales en cada clase de dibujo, y no se pasaba de una a otra superior, sin haber conseguido dos primeros premios. En la clase de "desnudo" nos quedábamos seis años, los que eran asignados a los pensionados, y cada mes era posible competir para adjudicarse premios. (p. 8, trad. propia $)^{13}$

\section{CONSIDERACIONES FINALES}

La dificultad de contestar a las preguntas acerca del Filoctetes refleja la complejidad de la definición de la pintura de Ciccarelli a partir de corrientes estilísticas decimonónicas precisas. Creemos que a ello contribuyeron de manera determinante las transformaciones radicales que afectaron a los tres países de actividad del pintor, los cuales, en los años de sus estadías, como mencionamos, estaban viviendo procesos y cambios políticos muy diferentes entre sí, los que repercutieron irremediablemente en las artes de cada territorio. De esta manera, Ciccarelli tuvo la difícil tarea de satisfacer gustos, necesidades y lenguajes muy variados, hecho que puede explicar la heterogeneidad temático-estilística de su producción, que, si bien ha sido en parte señalada por Pereira Salas, nosotros hemos querido definir gracias a la propuesta de una nueva catalogación de su obra por etapas.

Tras el análisis de la tela de la colección de las Gallerie del Museo di Capodimonte, junto con la recopilación de fuentes historiográficas de la época, como los escritos de Morelli, hemos informado sobre el sistema de enseñanza de la academia italiana y sobre la resonancia que el Filoctetes tuvo en el país, y hemos ofrecido nuevos datos acerca de los años menos investigados de la trayectoria artística del pintor napolitano.

Todo ello, para contribuir a superar la tradicional lectura de pintor frío y académico con que se ha etiquetado injustamente a Ciccarelli, en una

\footnotetext{
${ }^{13}$ Fuente original: L’ordine dei nostri studi, allora, era lo stesso di quello che si usa tenere ora; dalla stampa si passava al rilievo, e dalla statua al nudo ed alla pittura. Vi erano concorsi mensili in ogni classe di disegno, e non si usciva dall'una, per entrare all'altra superiore, senza avere avuto due primi premi. Nella classe del «nudo» si restava sei anni, quanti ne erano assegnati ai pensionati, e si poteva tutti i mesi concorrere ed avere premi.
} 
reductora tentativa de su inclusión en las categorías del estilo Neoclásico o Romántico.

Consideramos que su papel como iniciador de la sistematización de los estudios artísticos modernos en Chile a partir de la formación en dibujo, elemento compositivo sin duda protagonista en el Filoctetes abandonado, ha sido objeto de cierta tergiversación, y que ello se debió a las definiciones cambiantes que la historiografía del siglo pasado ha dado sobre la disciplina del dibujo, que abren un debate muy amplio acerca de la adhesión de Chile a los cánones artísticos establecidos por una cultura visual de raigambre italiana.

De hecho, creemos que el pintor tratado en estas páginas es la prueba de la necesidad de investigación acerca del intenso entramado de influencias artísticas entre Italia y Chile, que amplíen y profundicen acerca de las "fructíferas relaciones" que Sartor (1997, p. 57) teorizó.

\section{REFERENCIAS}

Accademia delle Belle Arti di Napoli. (1837). Lavori fatti in Roma da pensionati napoletani. Annali Civili del Regno delle due Sicilie XIII(25), 136-139.

Ciccarelli, A. (s.f.). Brasiliana Iconográfica, recuperado de https://www.brasilianaiconografica.art.br/obras/rel_content_id/19825/alessandro-ciccarelli

Ciccarelli, A. (s.f.). ICCD, recuperado de http://www.catalogo.beniculturali.it/ sigecSSU_FE/listaVersioniAutore.action?authority=true\&nomeBread $=$ Aut ori\&nomeScelto=Ciccarelli\%20Alessandro

Ciccarelli, A. (s.f.). SURDOC, recuperado de https://www.surdoc.cl/coleccion es? $\% 5$ B0\%5D=submit $\% 3 \mathrm{ABuscar} \&$ buscador $=$ ciccarelli

Ciccarelli, A. (s.f.). Warburg BCI, recuperado de http://warburg.chaa-unicamp. com.br/obras/view/8850

Álvarez Urquieta, L. (1928). La pintura en Chile. Colección Luis Álvarez Urquieta. Santiago: La Ilustración.

Bíndis, R. (1985). La pintura chilena: desde Gil de Castro hasta nuestros días. Santiago: Philips Chilena, Departamento de Publicidad.

Cinelli, N. (2019). "Bajo el bello cielo de Chile". Alessandro Ciccarelli, primer director de la Academia de Pintura en Santiago (siglo XIX). En M. A. Fernández Valle, C. López Calderón, y I. Rodríguez Moya (eds.). Pinceles y gubias del barroco iberoamericano (pp. 393-404). Santiago de Compostela: Enredars Andavira Editora.

Cruz de Amenábar, I. (2004). "La Atenas del Pacifico". Alejandro Cicarelli y el proyecto civilizador de las Bellas Artes en Chile republicano. Tiempos de América 11, 91-104.

Cruz, C. A. (1988). Homenaje a la Sociedad Filatélica de Chile. Colón, pintura 
de Cicarelli y el origen de nuestros primeros sellos. Chile Filatelico t. XXII (246), 206-209.

Dalbono, E. (1915). Scritti di Domenico Morelli. Commemorazione letta alla Real Accademia di Archeologia, Lettere e Belle Arti di Napoli il 25 di novembre del 1901. En B. Croce (ed.). La scuola napoletana di pittura nel secolo decimonono ed altri scritti d'arte (pp. 67-101). Bari: Laterza.

De la Maza, J. (2012). Duelo de pinceles. Ernesto Charton y Alejandro Ciccarelli. Pintura y enseñanza en el siglo XIX chileno. En F. Guzmán y J. M. Martínez (eds.). Vínculos artísticos entre Italia y América. Silencio historiográfico. VI Jornadas de Historia del Arte (pp. 218-220). Valparaíso: DIBAM, Museo Histórico Nacional, Facultad Artes Liberales de la Universidad Adolfo Ibáñez, Centro de Restauración y Estudios Artísticos CREA.

De La Maza, J. (2016). Alessandro Ciccarelli. En MNBA. En-Clave Masculino. Colección MNBA 2016. Santiago: DIBAM.

Don Alejandro Ciccarelli. (1885a). El Taller Ilustrado 19, 2.

Don Alejandro Ciccarelli. (1885b). El Taller Ilustrado 20, 2.

Fossi, G. (2015). Ottocento italiano. La pittura. Florencia: Giunti.

Freire, L. (1983). Um século de pintura: apontamentos para a história da pintura no Brasil de 1816-1916. Rio de Janeiro: Fontana.

Galaz, G. e Ivelic M. (2009). La pintura en Chile desde la colonia hasta 1981. Valparaíso: Ediciones Pontificia Universidad Católica de Valparaíso.

Lima, V. (2010). Alessandro Ciccarelli e a tela "Casamento por procuração da imperatriz D. Teresa Cristina”: um ensaio interpretativo. En A. Valle y C. Dazzi (eds.). Oitocentos - Arte Brasileira do Império à República (tomo 2, pp. 657-669). Rio de Janeiro: Seropédica.

Lima, V. (2012). Da Italia ao continente americano: Alessandro Cicarelli entre Brasil e Chile. En F. Guzmán y J. M. Martínez (eds.). Vínculos artísticos entre Italia y América. Silencio historiográfico. VI Jornadas de Historia del Arte (pp. 185-195). Valparaíso: DIBAM, Museo Histórico Nacional, Facultad Artes Liberales de la Universidad Adolfo Ibáñez, Centro de Restauración y Estudios Artísticos CREA.

Lira, P. (1902). Diccionario biográfico de pintores. Santiago: Imprenta Encuadernación y Litografía Esmeralda.

Lorenzetti, C. (1953). L’Accademia di Belle Arti di Napoli, 1752-1952. Firenze: Le Monnier.

Morelli, D. (1915). Filippo Palizzi e la scuola napoletana di pittura dopo il 1840. Discorso tenuto all'Accademia Reale il 21 giungno 1900. En B. Croce (ed.). La scuola napoletana di pittura nel secolo decimonono ed altri scritti d'arte (pp. 3-41). Bari: Laterza.

Pateras, T. (1872). Arte Contemporanea. Pittura Napolitana. L’Arte in Italia. Rivista mensile di Belle Arti año 4, 8, 116-120.

Pereira Salas, E. (1992). Estudios sobre la Historia del Arte en Chile Republicano. Santiago: Editorial Universitaria.

Plath, O. (2003). Origen y folclor de los juegos en Chile. Santiago: Grijalbo. 
Poppi, C. (1996). Pelagio Pelagi, Pittore. Dipinti dalle raccolte del Comune di Bologna. Bolonia: Electa.

Richter, M. y Valdivieso, C. (2012). La presencia del pintor italiano Alejandro Ciccarelli (ca. 1810-1879) en la Academia de Pintura de Chile: sus actividades directivas. En F. Guzmán y J. M. Martínez (eds.). Vínculos artísticos entre Italia y América. Silencio Historiográfico. VI Jornadas de Historia del Arte (pp. 185-195). Valparaíso: DIBAM, Museo Histórico Nacional, Facultad Artes Liberales de la Universidad Adolfo Ibáñez, Centro de Restauración y Estudios Artísticos CREA.

Ripamonti Montt, V. (2010). Academia de Pintura en Chile: sus momentos previos. Intus-Legere Historia 4(1), 127-153.

Romera, A. (1951). Historia de la Pintura en Chile. Santiago: Editorial del Pacífico.

Sartor, M. (1997). Le relazioni fruttuose. Arte ed artisti italiani nell'Accademia di San Carlos di Messico. Ricerche di Storia dell'Arte, 63, 7-34.

Zamorano, P. (2013). El discurso de Ciccarelli en la fundación de la Academia de Pintura de Chile (1849). Quiroga 4, 76-86. 